\title{
Marcelina Kałasznik*
}

\section{STAR, CELEBRITY, VIP - ZUM EINFLUSS DES ENGLISCHEN AUF DAS WORTFELD DER BEZEICHNUNGEN FÜR BEKANNTE PERSONEN IM DEUTSCHEN ${ }^{1}$}

Die Entlehnungen und ihr Einfluss auf die Nehmersprache werden unterschiedlich empfunden ${ }^{2}$. Zum einen wird die Meinung vertreten, dass sie die Lexik der jeweiligen Sprache bereichern. Zum anderen haben manche Sprachforscher und Sprachnutzer eine kritische und abweisende Einstellung Fremdwörtern gegenüber $^{3}$ (vgl. Yang 1990, S. 1). Sick (2009, S. 151) stellt in diesem Kontext Folgendes fest: „Fremdwörter, egal welcher Herkunft, werden zunächst mit Ehrfurcht und Respekt behandelt, manche Menschen fassen sie mit Samthandschuhen an, andere nur mit spitzen Fingern. Man ist im Allgemeinen froh, wenn man weiß, was sie bedeuten [...]. Doch je mehr man sich an sie gewöhnt, desto geringer werden die Berührungsängste. [...]“

In dem Beitrag wird versucht, auf die Frage der Beeinflussung des Wortschatzes der deutschen Sprache durch das Englische einzugehen, wobei auf einen besonderen Sinnbezirk der deutschen Lexik fokussiert wird. Im Zentrum des Interesses steht nämlich das Wortfeld der individuellen Bezeichnungen ${ }^{4}$ für öffentlich bekannte Personen ${ }^{5}$. Die Ziele, die dabei verfolgt werden, sind mit einigen Aspekten der Aufnahme und Integration der aus dem Englischen übernommenen

* Dr. Marcelina Kałasznik, Universität Wrocław, Institut für Germanische Philologie, Lehrstuhl für Deutsche Sprache, Pl. Nankiera 15b, 50-140 Wrocław. E-Mail: marcelina.kalasznik@uwr.edu.pl

${ }^{1}$ Der Beitrag ist eine überarbeitete Version des Referats, das auf der 24. Jahrestagung der Gesellschaft für Sprache und Sprachen (GeSuS) „Sprachen verbinden“ (22.-24.06.2016) präsentiert wurde.

2 Zur Debatte über Anglizismen im Deutschen, vgl. z.B. Miller (2003).

${ }^{3} \mathrm{Zu}$ Motiven für die Bekämpfung von Fremdwörtern im Deutschen, vgl. Lipczuk (2012).

${ }^{4}$ Die sog. personalen Kollektiva, z.B. Crème de la Crème, die auf Gruppen von bekannten Personen referieren, werden in diesem Beitrag aus Raumgründen außer Acht gelassen.

${ }^{5}$ Zum analysierten Wortfeld können folgende Lexeme gezählt werden: Berühmtheit, Celebrity, Diva, Größe, Idol, Ikone, Legende, Persönlichkeit, Prominente(r)/Promi, Star, Stern, VIP, wobei diese hier präsentierte Auflistung der Feldglieder ausbaufähig ist. Je nach Kriterien, die man bei der Auffindung der Außengrenzen des Feldes heranzieht, kann das Wortfeld um andere Lexeme ergänzt werden. 
lexikalischen Einheiten im Deutschen verbunden. Zuerst soll nämlich der Aspekt der semantischen Adaptation der analysierten Lexeme im Deutschen erörtert werden. Weiterhin ist beabsichtigt, zu ermitteln, inwieweit die aus dem Englischen in unveränderter morphologischer Form übernommenen Lexeme im Deutschen verbreitet sind, da die Verwendungsfrequenz der Anglizismen einen der Faktoren darstellt, der von ihrem Integrationsgrad in der Nehmersprache zeugt. Nicht zuletzt ist der Frage nachzugehen, wie oft die entlehnten Einheiten zu unmittelbaren Konstituenten für Wortbildungen im Deutschen werden. Diese kurz umrissenen Forschungsfragen sollten es einerseits erlauben, den Integrationsgrad der besprochenen Personenbezeichnungen im Deutschen zu ermitteln, und andererseits ihr jeweiliges Verhältnis zu deutschen Pendants zu präsentieren. Mit einem solch konzipierten Analyserahmen sollte außerdem das Ziel erreicht werden, über die rein linguistische Ebene hinauszugehen und auf kulturelle Aspekte aufmerksam zu machen, die der Entlehnung in diesem Bereich zugrunde liegen.

Das Korpus für diese Studie machen ausgewählte deutsche Pressetitel ${ }^{6}$ aus, die den Unterhaltungssektor der Zeitschriften für Frauen repräsentieren. Ergänzend werden auch exemplarisch Belege aus dem online verfügbaren DWDS (Digitales Wörterbuch der Deutschen Sprache) herangezogen.

\section{Zu Funktionen von Personenbezeichnungen}

Die hier zu besprechenden Anglizismen sind Personenbezeichnungen, die wie folgt definiert werden: ,[...] Benennungen für alle möglichen Existenzformen und Seinsweisen des Menschen; sie drücken aus, wie der Mensch den Menschen benennt, wie er sich und andere versteht, einschätzt und beurteilt.“ (Braun 1990, S. 167). Die Vielfalt der in jeder Sprache zur Verfügung stehenden Personenbezeichnungen erlaubt es also, den Menschen als Handlungssubjekt oder -objekt möglichst genau zu erfassen (vgl. Braun 1997, S. 145).

Vom linguistischen Gesichtspunkt aus stellen Personenbezeichnungen ein durchaus interessantes und ergiebiges Forschungsfeld dar, denn sie dienen nicht der bloßen Referenzfixierung, sondern es werden mit ihnen bestimmte Assoziationen verbunden, die eine große Bedeutung für das Bild der benannten Menschen und für die Rezeption eines Textes haben. Die Auswahl einer Personenbezeichnung aus der Palette von verschiedenen Nominationsalternativen weist zugleich auf eine Perspektive hin, aus der eine bestimmte Person betrachtet und auch bewertet wird, da die Personenbezeichnungen einen besonderen Beitrag durch ihr denotatives und konnotatives Potenzial leisten (vgl. Braun 1997, S. 145). Im Zusammenhang mit der Möglichkeit einer Selektion unterschiedlicher sprachli-

\footnotetext{
${ }^{6}$ Vgl. Quellenverzeichnis am Ende des Beitrags.
} 
cher Mittel, mithilfe derer auf dasselbe Referenzobjekt Bezug genommen werden kann, spricht man von Bezeichnungskonkurrenz oder Nominationskonkurrenz (vgl. Spieß 2011, S. 141). Die unterschiedlichen Bezeichnungen referieren somit auf dasselbe Objekt, wobei sie andere Bewertungen vermitteln können. Mit der Wahl einer konkreten Bezeichnungsalternative geht jeweils eine Evaluation einher (vgl. Spitzmüller/Warnke 2011, S. 141).

Die primäre Funktion analysierter Einheiten besteht in der Benennung von Personen. Es kann jedoch nicht außer Acht gelassen werden, dass mit personenbezeichnenden Appellativa auch kategorisiert, charakterisiert und bewertet wird. Herrmanns (1994, S. 55) versucht, auf Relationen von Personenbezeichnungen und sozialen Kategorien hinzuweisen, die die Funktionen der Personenbenennungen verdeutlichen. Herrmanns (1994, S. 55) unterscheidet nämlich erstens Personenbezeichnungen in Funktion von sprachlichen labels, also Etiketten, die für bestimmte Kategorien stehen und mit denen die Personen sozial verortet werden. Die Personenbezeichnung, konfrontiert mit Merkmalen einer Person, erlaubt es, sie als ein typisches oder untypisches Mitglied einer personalen Kategorie zu erfassen. Zweitens können Personenbezeichnungen in Funktion von Chiffren gebraucht werden, mit denen bestimmte Informationen verschlüsselt werden. Erst durch Verfolgung und Untersuchung der diskursiven Verwendung von Personenbezeichnungen können die dahinter versteckten Informationen entziffert werden. Drittens sind Personenbezeichnungen als Abbreviaturen zu betrachten, in denen komplexe Wissensbestände sozialen oder kulturellen Charakters gespeichert werden.

\section{Anglizismus - Zur Begriffsbestimmung}

Der Entlehnung liegt Sprachkontakt zugrunde, der folgendermaßen definiert werden kann: ,Sprachberührung oder gegenseitiges Aufeinanderwirken von Sprachen aufgrund kommunikativer Interaktionen von Sprechern unterschiedlicher Sprachen unter besonderen geographischen, historisch-politischen, kulturellen und sozialen Gegebenheiten mit erkennbaren Einflüssen von Sprachen aufeinander, die als Sprechgewohnheiten und u.U. bleibende Sprachveränderungen manifest werden“ (Lewandowski 1994, S. 1026f.). Das Resultat des Sprachkontakts sind lexikalische Entlehnungen sowie Interferenz- und Integrationsphänomene, wobei das Vokabular einer jeden Sprache - als am meisten fluider und offener Teil des Sprachsystems - gegen Änderungen unter fremdsprachlichem Einfluss besonders empfindlich ist. Die Übernahme des Fremdwortschatzes ist kein zufälliger Prozess, sondern er beruht auf kulturellen, politischen und ökonomischen Voraussetzungen (vgl. Braun 1997, S. 131). In der Sprachgeschichte lassen sich somit Epochen unterscheiden, in denen sich aus genannten Gründen vorherrschende Tendenzen in Bezug auf die Übernahme des Wortschatzes aus einer Sprache abzeichnen lassen. Aus den Untersuchungen, die darauf abzielen, die Präsenz der Anglizismen in der 
deutschen Sprache aus diachronischer Perspektive zu verfolgen, geht hervor, dass das Interesse am Englischen seit dem 17. Jh. allmählich ansteigt (vgl. z.B. Kovács 2009, S. 182). Das 18. Jh. ist die Zeit, in der relativ viele Anglizismen in die deutsche Sprache entlehnt wurden, wobei sie auf unterschiedliche Sachgebiete verteilt waren. Wie Eisenberg (2011, S. 48) feststellt, „[beginnt - M. K.] der Siegeszug des Englischen als Gebersprache um die Wende zum 19. Jhdt. und wurde seitdem so gut wie nicht verlangsamt, geschweige denn angehalten." Und als die Epoche des stärksten Einflusses sowohl des britischen als auch des amerikanischen Englischen gilt die Zeit nach 1945, so dass das Englische zur Zeit als die Hauptgebersprache für die moderne deutsche Sprache fungiert (vgl. Dargiewicz 2013, S. 51). ${ }^{7}$

In der Forschungsliteratur wird deutlich hervorgehoben, dass „Das Deutsche es einerseits mit Entlehnungen aus dem Englischen leicht [hat - M.K.], weil beide Sprachen historisch und typologisch eng verwandt sind. [...] Dasselbe gilt für die Wörter. Auch hier gibt es so viele Gemeinsamkeiten, dass ein Hin und Her zwischen den Sprachen ohne Schwierigkeiten möglich ist.“ (Eisenberg 2011, S. 55). Zum Thema der Anglizismen liegt bereits eine umfangreiche Literatur vor, wobei es keine Übereinstimmung darüber herrscht, welche Einheiten als Anglizismen anzusehen sind. In diesem Beitrag, der auf einen kleinen Teil des deutschen Wortschatzes begrenzt ist, wird die folgende Definition als ausschlaggebend angenommen, in der der Terminus Anglizismus breit aufgefasst wird: ,jede Erscheinung der deutschen Sprache, die auf Transferenz des Englischen zurückgeht. In dieser Verwendungsweise dient der Terminus als Oberbegriff für alle Varietäten des Englischen und schließt auch das innere Lehngut mit ein" (Busse 2011, S. 99, in: Dargiewicz 2013, S. 52).

\section{Anglizismen im Wortfeld der Bezeichnungen für bekannte Personen - Analyse des Materials}

Im Folgenden werden die Lexeme Celebrity, Star und VIP 8 im Hinblick auf ihre Semantik, Geläufigkeit und Wortbildungsproduktivität hin analysiert. Was die erste Forschungsfrage - die Bedeutung(sstruktur) der untersuchten Fremdwörter im Deutschen - angeht, wird davon ausgegangen, dass die Übernahme eines Lexems in ein anderes kommunikatives Umfeld die Änderung seiner Semantik bewirken kann. Somit wird versucht, festzustellen, ob der vollständige Bedeutungsumfang der lexikalischen Einheit ins Deutsche übernommen oder ob lediglich ein Teil der ursprünglichen Bedeutung in die Nehmersprache entlehnt wurde. Die weitere Analysetappe besteht darin, die Vorkommenshäufigkeit der Analyseeinheiten

${ }^{7}$ Aus Raumgründen kann hier kein fundierter Überblick über die Geschichte des Einflusses des Englischen auf die deutsche Sprache gegeben werden. Zu diesem Thema, vgl. z.B. Eisenberg (2011).

${ }^{8}$ Die Lexeme werden in alphabetischer Reihenfolge besprochen. 
darzustellen. Danach sollte aufgrund der Analyse der Wortbildungen mit den besprochenen Lexemen gezeigt werden, wie sich die „,Zwei Hauptwege der Erweiterung des Wortschatzes“ (Fleischer 1977, S. 64) - Wortbildung und Entlehnung - kreuzen.

Bei dem ersten Analyseschritt werden Wörterbücher der deutschen Gegenwartssprache und als Ergänzung Fremdwortlexika herangezogen. In weiteren Etappen der Analyse - bei der Ermittlung der Frequenz und Wortbildungsaktivität der Lexeme - werden grundsätzlich Beispiele aus dem Pressekorpus und dem DWDS präsentiert.

\subsection{Celebrity}

\subsubsection{Semantik}

Die lexikographische Erfassung des Lexems celebrity/Celebrity in Nachschlagewerken des Englischen und Deutschen lässt sich der nachstehenden Tabelle entnehmen.

Tabelle 1. Semantik des Lexems celebrity/Celebrity in Anlehnung an Wörterbücher der englischen und deutschen Gegenwartssprache

\begin{tabular}{|l|l|}
\hline \multicolumn{2}{|c|}{ Lexikographische Erfassung der Lexeme } \\
\hline \multicolumn{1}{|c|}{ celebrity, Pl. celebrities } & \multicolumn{1}{|c|}{ Celebrity, die, Pl. Celebritys/Celebrities } \\
\hline $\begin{array}{l}\text { CEL* (1992, S. 219): 'a celebrity is someone } \\
\text { who has become famous for something, } \\
\text { especially for something connected with acting } \\
\text { or show business' } \\
\begin{array}{l}\text { CD ONLINE**: } 1 \text {. 'someone who is famous, } \\
\text { especially in the entertainment business' } 2 .\end{array}\end{array}$ & $\begin{array}{l}\text { DUDEN ONLINE***: 'Berühmtheit, } \\
\text { 'the state of being famous' }\end{array}$ \\
\hline
\end{tabular}

* Die jeweilige Abkürzung der vollständigen Titel jedes Wörterbuchs ist nach bibliographischen Angaben im Literaturverzeichnis platziert.

** Vgl. http://dictionary.cambridge.org/dictionary/english/celebrity, Zugriff am 7.07.2016.

***Vgl. http://www.duden.de/rechtschreibung/Celebrity, Zugriff am 7.07.2016.

Das Substantiv celebrity hat im Englischen, wie es die Wörterbuchdefinitionen bestätigen, zwei Bedeutungen. Es bezieht sich erstens auf Ruhm/Popularität und zweitens auf Personen, die einen Ruhm erlangt haben. Die Analyse der Einträge aus deutschen Nachschlagewerken verdeutlicht zunächst, dass die Entlehnung kein völlig integrierter und konventionalisierter Anglizismus ist. Die bescheidene lexikographische Erfassung des Lexems in den meisten derzeit zugänglichen Wörterbüchern der deutschen Gegenwartssprache zeugt von dessen Neologismus-Status. Die interlinguale Betrachtung des Bedeutungsumfangs 
des Lexems zwischen abgebender und aufnehmender Sprache lässt Celebrity im Deutschen als einen Anglizismus mit Bedeutungsverengung ${ }^{9}$ bezeichnen, da es in der deutschen Sprache nur in der personalen Bedeutung gebraucht wird. Die nicht-personale semantische Variante ist in der Bedeutungsstruktur des Lexems im Deutschen nicht vorhanden.

\subsubsection{Frequenz}

Das Pressekorpus liefert keine Belege für den Gebrauch des Lexems. Die Durchsuche der DWDS-Kernkorpora 20 und 21 ergibt keine Treffer. Bei der Suche im Korpus der Zeitung „Die Zeit“ werden 172 Belege erzielt. Die Aufteilung der Belege im Hinblick auf ihre Funktion wird im nachfolgenden Diagramm illustriert.

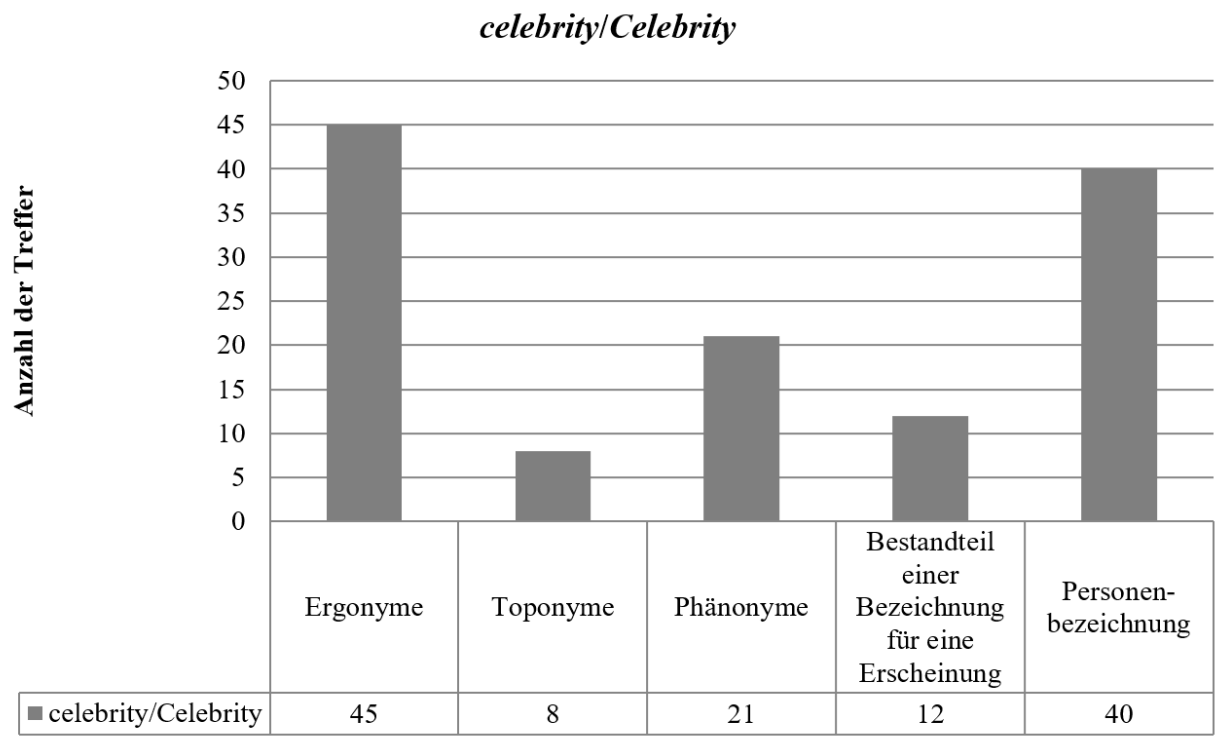

Diagramm 1. Gebrauch des Lexems celebrity/Celebrity im Deutschen

Das oben angeführte Diagramm sollte veranschaulichen, dass das Lexem celebrity/Celebrity am häufigsten im DWDS-Subkorpus als Komponente von Eigennamen vorkommt. Dabei sind die folgenden Klassen von Eigennamen vertreten: Ergonyme: Titel von Reality-Shows: ,The Celebrity Apprentive "10 , ,Celebrity Big Brother “, „Best Celebrity Home”; Titel von Büchern: „,How to be a Celebrity”;

${ }^{9}$ Der Terminus Anglizismus mit Bedeutungsverengung wurde hier in Anlehnung an einen bekannten kroatischen Anglisten Filipović (1988) verwendet.

${ }^{10}$ Die Schreibweise des Eigennamens mit und ohne Anführungszeichen wird dem jeweiligen Kontextbeispiel entnommen. Die Schreibweisen werden somit nicht geändert, sondern direkt übernommen. 
Titel von Filmen: „Celebrity “; Titel von CD-Alben: Celebrity Skin; Bezeichnungen für Schiffe: ,Celebrity Solstice“, „Celebrity Reflection”, Celebrity Eclipse; Unternehmensnamen: Celebrity Management Group; Toponyme: Celebrity Centers; Phänonyme: Das Jerry Ford Celebrity Cup, „Celebrity Walk“, Celebrity Charity Auction, Burns Sport Celebrity, Most powerful Celebrity ${ }^{11}$. Das Lexem celebrity/Celebrity tritt ebenfalls als Bestandteil von Bezeichnungen für Phänomene aus dem Bereich der Medienwissenschaft auf, z.B. Celebrity Placement, celebrity culture, Celebrity Performance. Dem Substantiv kommt auch die Funktion einer eigenständigen Personenbezeichnung zu, wobei es in der Anfangsphase seiner Verwendung kleingeschrieben wird. Die großgeschriebene Variante scheint sich erst gegen das Jahr 2007 langsam durchzusetzen.

\subsubsection{Wortbildungsproduktivität}

Sowohl im Pressekorpus als auch in den DWDS-Korpora wurden ausschließlich Komposita im substantivischen Bereich mit der Konstituente Celebrity als Bestimmungsglied verzeichnet, z.B. Celebrity-Kult, Celebrity-Fotos (Br 16/2012, S. 56), Celebrity-Liebling (LS 30/2012, S. 17), Celebrity-Trainer (LS 32/2012, S. 11). Die kleine Anzahl der Beispiele scheint den Status der Einheit als Neologismus zu bestätigen. Bei den verzeichneten Komposita handelt es sich teilweise um die sog. Hybridbildungen ${ }^{12}$, d.h. Kombinationen aus indigenen und exogenen Elementen. Zu beachten ist, dass unter den zusammengestellten Beispielen, die das Vorkommen des Lexems Celebrity als unmittelbare Konstituente in Wortbildungen der deutschen Sprache belegen, das Substantiv in Funktion der Basis nicht registriert wurde. Auf dem Lexem Celebrity als Erstkonstituente liegt also kein Informationsschwerpunkt, sondern es wird dazu eingesetzt, die Bedeutung der Basis in bestimmter Hinsicht zu determinieren, zu modifizieren und zu konkretisieren (vgl. Bizukojć 2011, S. 15).

\subsection{Star}

\subsubsection{Semantik}

Das Substantiv star weist im Englischen viele Bedeutungsvarianten auf, von denen die meisten als metaphorische Verschiebungen von der grundsätzlichen Bedeutung im Sinne von Himmelskörper/Gestirn ausgelegt werden können. Mit der

${ }^{11}$ Aus Raumgründen können hier nicht alle Beispiele, die die einzelnen unterschiedenen Subkategorien repräsentieren, angeführt werden.

${ }_{12}$ Zur umfangreichen und ausführlichen Darstellung von Hybridbildungen im Deutschen, vgl. Dargiewicz (2013). 
personalen Bedeutungsvariante wird im Englischen auf Personen referiert, die bekannte und erfolgreiche Sänger, Schauspieler oder Sportler sind. Betrachtet man die deutschen lexikographischen Definitionen, erweist sich zunächst, dass nur eine semantische Variante aus dem Englischen ins Deutsche übernommen wurde.

Tabelle 2. Semantik des Lexems star/Star in Anlehnung an Wörterbücher der englischen und deutschen Gegenwartssprache

\begin{tabular}{|c|c|}
\hline \multicolumn{2}{|c|}{ Lexikographische Erfassung der Lexeme } \\
\hline star, Pl. stars & Star, der, Pl. Stars \\
\hline $\begin{array}{l}\text { CD ONLINE': 1.'a very } \\
\text { large ball of burning gas in } \\
\text { space that is usually seen from } \\
\text { the earth as a point of light in } \\
\text { the sky at night'; } 2 \text {. 'a very } \\
\text { famous, successful, and im- } \\
\text { portant person, especially } \\
\text { a performer such as a musi- } \\
\text { cian, actor, or sports player'; } \\
\text { 3. 'a symbol with four or more } \\
\text { points', 4. used to show how } \\
\text { good a restaurant, hotel, etc. } \\
\text { is'; } 5 \text {. an asterisk (= a symbol } \\
* \text { ); } 6 . \text { 'informal any planet or } \\
\text { other object in the sky thought } \\
\text { of in astrology as influencing } \\
\text { a person's luck'; }\end{array}$ & $\begin{array}{l}\text { DUDEN ONLINE**: 1. (Theater, Film) 'gefeierter, berühmter } \\
\text { Künstler/gefeierte, berühmte Künstlerin'; } \\
\text { 2. 'jemand, der auf einem bestimmten Gebiet Berühmtheit er- } \\
\text { langt hat'; } \\
\text { FWL (2004, S. 948): 'gefeierte Persönlichkeit (von Film, Bühne } \\
\text { oder Sport)'; } \\
\text { MFL (2005, S. 533): 'beliebter (Film)Schauspielerin, berühmte } \\
\text { Persönlichkeit des Showgeschäfts'; (allg.) 'Publikumsliebling'; } \\
\text { AngW3 (1996, S. 1401-1408): 1. 'erfolgreiche populäre und be- } \\
\text { rühmte Persönlichkeit auf dem Gebiet der unterhaltenden Kunst, } \\
\text { meist von Schauspielern in Film und Fernsehen, Musikern etc., } \\
\text { die als Idol angesehen und gefeiert werden'; 2. 'Person, die auf } \\
\text { einem bestimmten Gebiet außerhalb der Unterhaltungsindustrie, } \\
\text { z.B. im Sport oder in der Politik, durch herausragende Leis- } \\
\text { tungen Berühmtheit erlangt hat, im Mittelpunkt des Interesses } \\
\text { steht'; 3. 'bekannte, häufig »berühmt-berüchtigte« Person die im } \\
\text { Mittelpunkt des Interesses steht oder stehen möchte, sich in den } \\
\text { Vordergrund drängt, die Hauptrolle spielen will, sich (zu sehr) } \\
\text { um öffentliches Ansehen bemüht'; 4. 'herausragende, außerge- } \\
\text { wöhnliche Sache, die bes. attraktiv und beliebt ist, Aufmerksam- } \\
\text { keit erregt' }\end{array}$ \\
\hline
\end{tabular}

* Vgl. http://dictionary.cambridge.org/dictionary/english/star, Zugriff am 7.07.2016.

** Vgl. http://www.duden.de/rechtschreibung/Star_Kuenstler_Beruehmtheit, Zugriff am 7.07.2016.

Die Entlehnung nur der personalen Bedeutungsvariante aus dem vollen Bedeutungsumfang des Wortes lässt feststellen, dass es in diesem Falle zur interlingualen Bedeutungsverengung (vgl. Filipović 1988) gekommen ist. Es kann jedoch festgestellt werden, dass die eine semantische Variante - Personenbezeichnung Star -, die in die deutsche Sprache entlehnt wurde, im Deutschen eine Bedeutungserweiterung erfahren hat. Die Bedeutungserweiterung besteht nämlich darin, dass das Lexem im Deutschen häufig mit einer negativen konnotativen Komponente verbunden ist. Dies bezeugt die dritte Bedeutungserläuterung aus dem AngW3, in der die Bedeutungsverschlechterung dieser Personenbezeichnung deutlich zum Ausdruck kommt. Wie man den weiterführenden Erklärungen in diesem Wörterbuch entnehmen kann, fehlt dem englischen Vorbild diese pejorative semanti- 
sche Nuance. Die Bezeichnung kann im Deutschen in bestimmten Kontexten als Anzeichen des Sarkasmus und der Ironie angesehen werden, die bekannten und launischen Personen gegenüber geäußert werden.

\subsubsection{Frequenz}

Das Lexem Star wird im Pressekorpus 814-mal nachgewiesen, wobei die Mehrheit der Beispiele (754) die Verwendung des Lexems als Konstituente von personenbezeichnenden Komposita illustriert. Im DWDS-Kernkorpus findet man zwar lediglich 36 Beispiele, aber in dem Subkorpus der Zeitung „Die Zeit“ beläuft sich die Anzahl der Beispiele schon auf über 21000 (genau 21 493).

Die Verbreitung des Lexems Star im Deutschen, hat Yang (1990) anhand von Texten aus dem Nachrichtenmagazin „Der Spiegel“"verfolgt. Die Zahlangaben zur Vorkommenshäufigkeit des Lexems zeigen, dass seit den 1950er Jahren die Gebrauchshäufigkeit der Personenbezeichnung Star in dieser Zeitschrift allmählich ansteigt $^{13}$. In der von Erkenbrecher (vgl. 2006, S. 19) durchgeführten Umfrage zu beliebtesten und am häufigsten benutzten Anglizismen im Deutschen erscheint die Personenbezeichnung Star an der 10. Stelle (vgl. Kovács 2009, S. 181). Die angeführten Zahlangaben aus relativ aktuellen Quellen sowie die Ergebnisse der ein wenig älteren Untersuchungen lassen somit vermuten, dass sich die Bezeichnung im Deutschen eingebürgert hat.

\subsubsection{Wortbildungsproduktivität}

Die vollständige Adaptation des Lexems kann auch mit der fast unüberschaubaren Anzahl von Wortbildungen nachgewiesen werden, in denen die Konstituente Star vorkommt, wobei hier Substantive - Produkte der Ableitung und Komposition - und adjektivische Zusammensetzungen vertreten sind. Unter den substantivischen Derivaten sind die folgenden zu verzeichnen: Starlet (AngW3 1996, S. 1408, WDS 1997, S. 871, FWL 2004, S. 948, ÜAng 2007, S. 215) und Startum (AngW3 1996, S. 1402). Das erstere gehört semantisch gesehen ebenfalls Personenbezeichnungen an und referiert auf junge und ehrgeizige Künstlerinnen - vor allem aus der Filmbranche, die sich in dem Beruf erst versuchen. Bei der Personenbezeichnung handelt es sich ebenfalls um eine direkte Entlehnung vom englischen gleich lautenden und gleich bedeutenden starlet. Die Tatsache, dass das Lexem in zahlreiche Wörterbücher der deutschen Sprache aufgenommen wurde, zeugt von der völligen Integration des Lexems in die deutsche Sprache. Dies trifft auf das Lexem Startum - die Bezeichnung für Zustand der Popularität - nicht ganz zu, das lediglich im Anglizismen-Wörterbuch verzeichnet ist.

${ }^{13}$ Yang (vgl. 1990, S. 37) gibt folgende Zahlen an: im Jahre 1950 - 21 Belege, im Jahre 1960 23 Belege, im Jahre 1970 - 53 Belege, im Jahre 1980 - 60 Belege. 
Zahlenmäßig überwiegt im substantivischen Bereich jedoch die Komposition, wobei sich hier eine dem Lexem Celebrity konträre Tendenz abzeichnet, dass die Konstituente Star insbesondere als Basis - und nicht als Bestimmungswort - vorkommt, z.B. TV-Star (C 30/2012, S. 15, C 30/2012, S. 56, C 31/2012, 13, C 31/2012, S. 23, C 31/2012, S. 35, C 31/2012, S. 36, C 32/2012, S. 10, C 32/2012, S. 28, C 39/2012, S. 54, C 41/2012, S. 59, C 32/2012, S. 55, C 33/2012, S. 20, C 33/2012, S. 29, C 34/2012, S. 10, C 34/2012, S. 27, C 35/2012, S. 7, C 35/2012, S. 13 , C 36/2012, S. 18, C 36/2012, S. 34, C 37/2012, S. 52, C 39/2012, S. 5, C 39/2012, S. 17, C 39/2012, S. 46, C 39/2012, S. 57, C 40/2012, S. 5, C 42/2012, S. 18, C 46/2012, S. 82, C 46/2012, S. 57, C 46/2012, S. 24 , C 46/2012, S. 14, C 46/2012, S. 14-15, C 45/2012, S. 57, C 45/2012, S. 31, C 45/2012, S. 29, C 45/2012, S. 26, C 44/2012, S. 26, C 44/2012, S. 22-23, C 43/2012, S. 32-33, C 50/2012, S. 52, C 49/2012, S. 21, C 49/2012, S. 4, C 48/2012, S. 54, C 47/2012, S. 20-21, C 48/2012, S. 54, 38/2012, S. 48, OK $38 / 2012$, S. 25 , OK 51/2012, S. 25, OK 50/2012, S. 10 , OK 45/2012, S. 66, OK 44/2012, S. 70), Soap-Star (C 30/2012, S. 15, C 30/2012, S. 34, C 33/2012, S. 9 , C 47/2012, S. 6, F 16/2012, S. 11), Comedy-Star (C 30/2012, S. 5, C 46/2012, S. 32), Castingstar (C 31/2012, S. 28, C 37/2012, S. 17, C 38/2012, S. 3), Kinostar (C 32/2012, S. 9, C 32/2012, S. 54, C 33/2012, S. 4, C 35/2012, S. 34, C 43/2012, S. 54, C 48/2012, S. 11)/Kino-Star (C 40/2012, S. 54, C 41/2012, S. 34, C 46/2012, S. 52, C 47/2012, S. 59, C 47/2012, S. 48), Doku-Star (C 32/2012, S. 30). Auf diese Art und Weise entstehen somit weitere Personenbezeichnungen, die die Referenzfiguren - Stars - im Hinblick auf folgende Merkmale näher spezifizieren: Wirkungsbereich, z.B. TV-Star, Zugehörigkeit zu einem Sender, Fernsehprogramm usw., z.B. RTL-Star, ,Bauer sucht Frau “-Star, Qualität, z.B. Superstar, Alter, z.B. Kinderstar, Herkunft, z.B. US-Star. Die Zusammensetzungen, in denen die Konstituente Star als Erstglied vorkommt, sind vorwiegend Personenbezeichnungen, die einen aufwertenden Charakter haben, z.B. Star-Paar (C 30/2012, S. 17), Star-Moderator (C 30/2012, S. 21), Star-Designer (C 30/2012, S. 29), Star-DJ (C 32/2012, S. 8), Starkoch (C 32/2012, S. 62), Star-Designer usw. In diese Gruppe reihen sich allerdings auch solche Bezeichnungen ein, wie etwa Star-Leben (C 33/2012, S. 24), Star-Power (C 34/2012, S. 29, C 38/2012, S. 80), Star-Style (C 34/2012, S. 54, C 39/2012, S. 54, C 40/2012, S. 54), Star-Appeal (Br 23/2012, S. 71), Star-Allüren (C 37/2012, S. 7) und Gegenstandsbezeichnungen, z.B. Star-Parfüms (C 32/2012, S. 38).

Im Pressekorpus wurden zwei adjektivische Kompositionen mit der Komponente Star belegt, nämlich stargespickt (OK 48/2012, S. 69) und starbesetzt (OK 35/2012, S. 80), die im Hinblick auf ihre Frequenz jedoch unterrepräsentiert zu sein scheinen ${ }^{14}$.

\footnotetext{
${ }^{14}$ Zur Wortfamilie des Lexems Star, vgl. Kałasznik (2014).
} 


\subsection{VIP}

\subsubsection{Semantik}

Aufgrund des interlingualen Vergleichs des Bedeutungsumfangs des Substantivs im Englischen und im Deutschen kann festgestellt werden, dass es sich bei der Bezeichnung VIP um einen Anglizismus mit Null-Extension ${ }^{15}$ handelt, d.h. mit gleicher Semantik in der Geber- und Nehmersprache.

Tabelle 3. Semantik des Lexems VIP in Anlehnung an Wörterbücher der englischen und deutschen Gegenwartssprache

\begin{tabular}{|l|l|}
\hline \multicolumn{2}{|c|}{ Lexikographische Erfassung der Lexeme } \\
\hline \multicolumn{1}{|c|}{ VIP, PI. VIPs } & \multicolumn{1}{|c|}{ VIP, der od. die, PI. VIPs/VIP } \\
\hline $\begin{array}{l}\text { CD ONLINE': 'a very important person; } \\
\text { a person who is treated better than ordinary } \\
\text { people because they are famous or influential } \\
\text { (= they have a lot of influence) in some way'; }\end{array}$ & $\begin{array}{l}\text { DUDEN ONLINE' ' wichtige Persönlichkeit } \\
\text { [mit Privilegien]'; }\end{array}$ \\
$\begin{array}{l}\text { WDS (1997, S. 1006): 'wichtige, bekannte } \\
\text { given better treatment than ordinary people } \\
\text { because they are famous, influential, or } \\
\text { important'; }\end{array}$ & $\begin{array}{l}\text { ÜAng (2007, S. 238): 'sehr wichtige oder } \\
\text { prominente Persönlichkeit'; }\end{array}$ \\
\hline
\end{tabular}

* Vgl. http://dictionary.cambridge.org/dictionary/english/vip?q=VIP, Zugriff am 7.07.2016.

** Vgl. http://www.duden.de/rechtschreibung/VIP, Zugriff am 7.07.2016.

\subsubsection{Frequenz}

Die Abbreviation VIP wird im Pressekorpus 24-mal nachgewiesen, wobei sie in 23 Beispielen als Kompositionskonstituente vorkommt. In den Korpora wird das Lexem relativ selten vertreten. Die Suche im Kernkorpus 20 erzielt 21 Treffer, im Kernkorpus 21 keine Ergebnisse und in dem Subkorpus der Zeitung „Die Zeit“ 9 Vorkommnisse. Die Verwendung der entlehnten Bezeichnung VIP im Deutschen ist, wie das von dem DWDS-Tool Wortverlauf generierte Schema zeigt, in den 1990er Jahren deutlich gestiegen, und manifestiert sich vorwiegend in Pressetexten. Die Zahlangaben zeugen jedoch davon, dass die Bezeichnung VIP im Deutschen weiterhin selten geblieben ist.

\footnotetext{
${ }^{15}$ Der Terminus geht auf Filipović (1988) zurück.
} 


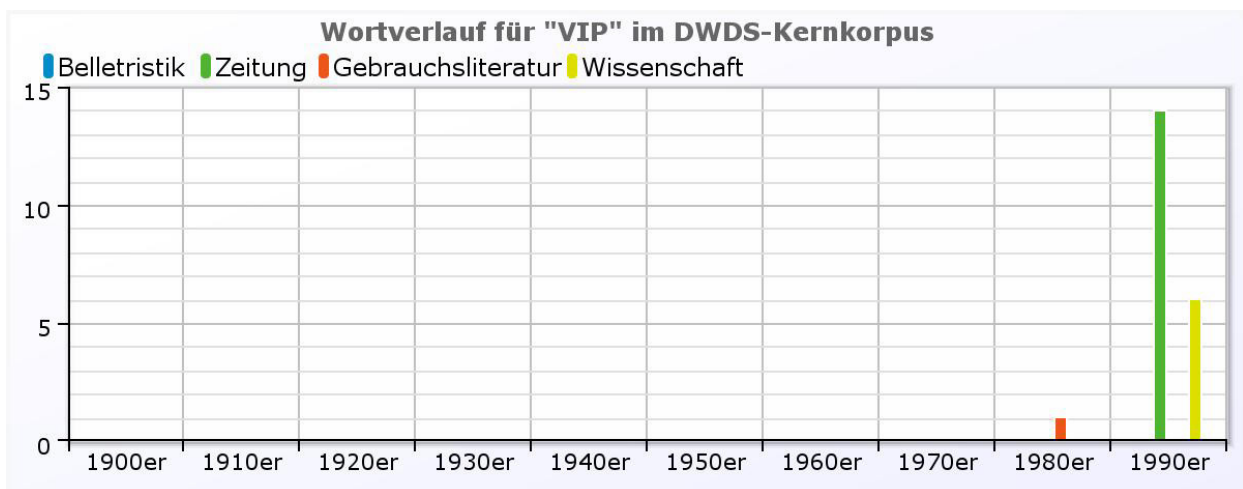

Schema 1. Quelle: DWDS; Zugriff 16.06.2016.

\subsubsection{Wortbildungsproduktivität}

Im zusammengestellten Korpus wurden substantivische Komposita verzeichnet, in denen der Konstituente VIP ausschließlich die Rolle des Erstgliedes zukommt. Semantisch gesehen lassen sie sich in Personenbezeichnungen, z.B. VIP-Gast (AngW3 1996, S. 1666, OK 39/2012, S. 69), VIP-Kunde (AngW3 1996, S. 1666), VIP-Ladys (C 42/2012, S. 12-13), VIP-Stylistin (F 17/2012, S. 11), VIP-Damen (LS 32/2012, S. 12), VIP-Unterstützer (OK 50/2012, S. 75), VIP-Kollegen (C 35/2012, S. 11), VIP-Wächter (C 44/2012, S. 10), Gegenstandsbezeichnungen, z.B. VIP-Armband (F 18/2012, S. 150), VIP-Tickets (OK 40/2012, S. 70), VIP-Shuttle (OK 50/2012, S. 75), VIP-Paket (OK 40/2012, S. 46), Bezeichnungen für Räume, z.B. VIP-Lounge, VIP Lounge, Vip-Lounge (AngW3 1996, S. 1666, C 40/2012, S. 9), VIP-Clubs (OK 35/2012, S. 20), VIP-Tribüne (OK 47/2012, S. 8), VIP-Dancefloors (OK 42/2012, S. 75), VIP-Bereich (C 36/2012, S. 30, OK 43/2012, S. 75), Bezeichnungen für Dienstleistungen, z.B. VIP-Service (AngW3 1996, S. 1666), VIP-Flug (AngW3 1996, S. 1666), VIP-Wochenende (C 32/2012, S. 49), Bezeichnungen für Veranstaltungen, z.B. VIP-Party (C 33/2012, S. 15, C 40/2012, S. 14, OK 50/2012, S. 75), VIP-Konzert (F 16/2012, S. 5), VIP-Fotoshooting (F 21/2012, S. 135-136), VIPEvents (OK 37/2012, S. 80, OK 38/2012, S. 75, OK 40/2012, S. 75) und sonstige Bezeichnungen, z.B. VIP-Treatment (AngW3 1996, S. 1666) VIP-Status (AngW3 1996, S. 1666) gliedern.

In Komposita, die auf Personen Bezug nehmen, erfüllt das Determinans VIP eine aufwertende Funktion und verleiht der benannten Personenkategorie Prestige. In den Zusammensetzungen mit der Erstkonstituente VIP - semantisch gesehen Gegenstands- und Sachverhaltsbezeichnungen - lässt sich das Verhältnis zwischen dem Erstglied und der Basis in den meisten Fällen als persönliche 
Zweckbestimmung ${ }^{16}$ interpretieren und mit der Paraphrase 'etw. ist für jmdn. bestimmt' umschreiben, z.B. VIP-Lounge ('Die Lounge ist für VIPs bestimmt'). Es finden sich auch Beispiele, in denen die Konstituente VIP die Qualität des vom Grundwort bezeichneten Objekts sprachlich verbessert und betont, z.B. VIP-Konzert.

Morphologisch betrachtet repräsentiert ein beträchtlicher Teil der Nominalkomposita den Typus der Hybridbildungen, z.B. VIP-Bereich, VIP-Flug, VIP-Wochenende. Viele Beispiele sind jedoch Zusammensetzungen, die nach dem englischen Vorbild im Deutschen gebildet wurden und aus zwei Anglizismen bestehen, z.B. VIP-Shuttle, VIP-Service, VIP-Fotoshooting.

Außerdem werden in den Wörterbüchern der Anglizismen Beispiele für adjektivische Derivate wie vipig (AngW3 1996, S. 1666) und VIPy (ÜAng 2007, S. 238) verzeichnet, die jedoch weder im Pressekorpus noch in den DWDS-Korpora nachgewiesen sind.

\section{Schlussfolgerungen}

Die drei analysierten Lexeme unterscheiden sich deutlich im Hinblick auf deren Gebrauch und Verbreitung in der deutschen Sprache.

Während Star als ein zentrales Lexem im Wortfeld der Bezeichnungen für bekannte Personen gilt und als ein vollintegrierter Anglizismus in der deutschen Sprache betrachtet werden kann, was seine Frequenz als selbständige Personenbenennung und seine Fähigkeit, Bestandteil neuer Wortbildungsprodukte zu werden, beweist, sind die Bezeichnungen Celebrity und VIP im Deutschen zur Zeit nicht so oft repräsentiert. Im Hinblick auf die Semantik der untersuchten Lexeme im zweisprachigen, englisch-deutschen Vergleich lässt sich konstatieren, dass die Personenbezeichnungen Celebrity und Star bei der Übernahme aus dem Englischen ins Deutsche eine Bedeutungsverengung erfahren haben, während die Denotation des Substantivs VIP in beiden Sprachen gleich ist.

Im Deutschen gibt es relativ viele andere Bezeichnungen, die dazu dienen, auf bekannte Personen Bezug zu nehmen. Manche von ihnen scheinen 1-1-Entsprechungen zu den hier besprochenen Anglizismen zu sein, z.B. Berühmtheit, Prominente(r) zu Celebrity oder Stern zu Star. Offensichtlich werden die indigenen deutschen Lexeme im medialen Diskurs auch gebraucht, wobei sie - das Lexem Prominente $(r)$ ausgenommen - im Vergleich mit den englischen seltener vorkommen. In diesem Sinne kann interessant sein, den Motiven für die Übernahme der Einheiten nachzugehen, da es sich dabei um keine Entlehnungen handelt, die aus sachlichen Gründen - zur Schließung einer lexikalischen Lücke

${ }^{16}$ Zur semantischen Typologie der Relationen zwischen den Konstituenten in Nominalkomposita, vgl. Bizukojć (2011). 
in diesem spezifischen Wortfeld - übernommen wurden. Es gibt verhältnismäBig viele Gründe ${ }^{17}$ für die Aufnahme von Anglizismen in die deutsche Sprache, von denen im Lichte des Analysegegenstandes der Ausdruck von „American Way of Life" besonders hervorgehoben werden sollte (vgl. Yang 1990, S. 2). Unter der Formulierung sind unterschiedliche Themenbereiche zu subsumieren, z.B. Bezeichnungen für Stile, Stilrichtungen und -aspekte der Rockmusik, Bezeichnungen für Kleidung, Speise- und Getränkebezeichnungen, Bezeichnungen aus dem Sport-, Film-, Fernsehbereich, Bezeichnungen für kosmetische Erzeugnisse und Teile oder Phänomene des gesellschaftlichen Lebens (vgl. Yang 1990, S. 2). In diesem Kontext können die Prozesse der Globalisierung nicht außer Acht gelassen werden, die auf unterschiedlichen Gebieten vor sich gehen, auch im Bereich der Kommunikation, des Lebensstils und der Werte. Damit kann die Übernahme der Bezeichnung Star aus dem Englischen ins Deutsche geklärt werden, da die große Popularität der Personenbenennung mit Erfolgen der US-Filmindustrie zusammenhängt. Die Verfolgung ihres Gebrauchs zeigt auch, dass sie in der Anfangsphase vorwiegend in Bezug auf Schauspieler angewendet wurde und erst in der weiteren Entwicklung ihre Extension auch auf Vertreter anderer Berufe ausgedehnt wurde, die erfolgreich sind. Ähnlich kann die Übernahme des Lexems Celebrity und seine langsame Adaptation im Deutschen erläutert werden. Mit dieser Bezeichnung wird im Englischen nämlich ein neues Phänomen benannt, das darin besteht, dass Menschen ohne einen besonderen Grund bekannt werden ${ }^{18}$. Diese Erscheinung, in Medien präsent zu sein und dadurch Bekanntheit zu erlangen, ohne große Leistungen zu erbringen und Erfolge zu feiern, entstammt der amerikanischen Medienlandschaft. Der sprachliche Mehrwert der Anglizismen, insbesondere in der Unterhaltungspresse, in der bekannte Personen einen eigenen Nachrichtenwert begründen, sollte somit darin bestehen, dass sie die Vorrangstellung der angloamerikanischen Kultur (vgl. Dargiewicz 2013, S. 53) in dem Bereich der Entwicklung der Medien und des öffentlich präsentierten Lebensstils zum Ausdruck bringen.

Der Verwendung der analysierten Bezeichnungen liegen ebenfalls stilistische Faktoren, Textsorte und kommunikative Situation zugrunde. Es handelt sich hier nämlich um mediale Texte, die das Thema des Show-Business aufgreifen, also vor allem unterhaltend und locker sein sollten. Die entlehnten Personenbezeichnungen oder Bezeichnungen, die auf Bedarf eines konkreten medialen Textes gebildet werden, haben einen besonderen Unterhaltungswert und machen den Text auffälliger, fesselnder und interessanter (vgl. Braun 1997, S. 147). Mit den Anglizismen können somit diese angestrebte Unterhaltung und

${ }_{17} \mathrm{Zu}$ anderen Gründen für die Übernahme von Anglizismen, vgl. Yang (1990).

${ }^{18}$ In diesem Sinne kann man auf die erste aber bis heute wiederholte Definition von Celebrity hingewiesen werden, in der die mit dieser Bezeichnung benannten Personen „ihrer allgemeinen Bekanntheit wegen [bekannt sind - M.K.]“. (Boorstin 1964, S. 39) 
Lockerung auf der sprachlichen Ebene erreicht werden, ohne dass die Kommunikation beeinträchtigt wird. Außerdem verleihen die Anglizismen den Texten Sozialkolorit und zeugen von Internationalität. Die internationale Dimension der Texte, die mithilfe von Anglizismen erzeugt wird, ist wiederum Ausdruck der Weltoffenheit.

In diesem spezifischen Wortfeld der Bezeichnungen für bekannte Personen kann der Einsatz von Anglizismen auch als ein Spiel mit dem Eskapismusverlangen der Leserinnen betrachtet werden (vgl. Dargiewicz 2013, S. 121), die sich sicherlich in die ferne, exotische, luxuriöse und unbekannte Welt der Stars und Celebrities entführen ließen.

Resümierend ist zu konstatieren, dass „Fremdwörter, darunter insbesondere Anglizismen, auch die nicht völlig in Laut und Schrift an die jeweilige Nehmersprache angepassten, daraus nicht wegzudenken [sind - M.K.], soweit sie eine lexikalische Lücke schließen bzw. stilistische Funktionen wahrnehmen, z.B. neue Denotate benennen resp. stilistisch markierte Schattierungen zum Ausdruck bringen." (Sadziński 2014, S. 169). Diese Feststellung scheint auf die hier besprochenen Lexeme im vollen Ausmaß zuzutreffen.

\section{QELLENVERZEICHNIS}

Brigitte, Nr. 16-26, Jahrgang 2012. [Br]

Closer, Nr. 30-50, Jahrgang 2012. [C]

Freundin, Nr. 16-26, Jahrgang 2012. [Fr]

Life\&Style, Nr. 30-32, Jahrgang 2012. [LS]

OK!, Nr. 35-50, Jahrgang 2012. [OK]

\section{VERZEICHNIS DER WÖRTERBÜCHER}

Anglizismen-Wörterbuch. Der Einfluss des Englischen auf den deutschen Wortschatz nach 1945

(1996). Begründet von Carstensen, B., fortgeführt von Busse, U. Bd. 3. Berlin. New York. [AngW3]

Cambridge Dictionary, http://dictionary.cambridge.org/[CD ONLINE]

Collins Cobild English Language Dictionary (1992). London. [CEL]

DUDENR (2006): Die deutsche Rechtschreibung. Mannheim. Leipzig. Wien. Zürich. [DudenR]

Duden Online, www.duden.de [DUDEN ONLINE]

Pogerell R., Schröder M. (Hrsg.) (2007): Wörterbuch überflüssiger Anglizismen. Paderborn. [ÜAng]

Sinclar J. (Hrsg.) (1992): Collins Cobuild English Language Dictionary. London. [CCEL]

Timm F. (Hrsg.) (2005): Das moderne Fremdwörterlexikon. Unbekannte Begriffe schnell verstehen und sicher anwenden. Köln. [MFL]

Wahrig (1997): Wörterbuch der deutschen Sprache. Wiesbaden. [WDS]

Wahrig-Burfeind R. (Hrsg.) (2004): WAHRIG Fremdwörterlexikon. Gütersloh. [FWL] 


\section{LITERATURVERZEICHNIS}

Bizukojć K. (2011), Neue Nominalkomposita in deutschen Newsletter-Texten, Frankfurt am Main.

Boorstin D. (1964), Das Image oder Was wurde aus dem amerikanischen Traum? Reinbeck bei Hamburg.

Braun P. (1990), Personenbezeichnungen. Der Mensch in der deutschen Sprache. In: Muttersprache 100, S. 167-191.

Braun P. (1997), Personenbezeichnungen. Der Mensch in der deutschen Sprache, Tübingen.

Busse U. (2011), Anglizismen - Versuch einer Bestandsaufnahme. In: Aptum. Zeitschrift für Sprachkritik und Sprachkultur. 7. Jahrgang, Bremen, S. 98-120.

Dargiewicz A. (2013), Fremde Elemente in Wortbildungen des Deutschen: Zu Hybridbildungen in der deutschen Gegenwartssprache am Beispiel einer raumgebundenen Untersuchung in der Universitäts- und Hansestadt Greifswald, Frankfurt am Main u.a.

Eisenberg P. (2011), Das Fremdwort im Deutschen, Berlin/New York.

Erkenbrecher Ch. (2009), Anglicisms in German. How many prominent Anglicisms are actually Pseudo-Anglicisms? Unter: http://www1.ku-eichstaett.de/SLF/EngluVglSW/schule32.pdf.

Filipović R. (1988), Teorija jezika u kontaktu (Theory of Languages in Contact), Zagreb.

Fleischer W. (1977), Entlehnung und Wortbildung in der deutschen Sprache der Gegenwart. In: Müller, P. O. (Hrsg.) (2005), Fremdwortbildung. Theorie und Praxis in Geschichte und Gegenwart, Frankfurt am Main, S. 63-76.

Hermanns F. (1994), Linguistische Anthropologie. Skizze eines Gegenstandsbereiches linguistischer Mentalitätsgeschichte. In: Busse D., Hermanns F., Teubert W. (Hrsg.): Begriffsgeschichte und Diskursgeschichte. Methodenfragen und Forschungsergebnisse der historischen Semantik, Opladen, S. 29-59.

Kałasznik M. (2014), ,,Im Mittelpunkt des öffentlichen Interesses stehend“-Eine Skizze der Wortfamilie ,Star" und ,,gwiazda“. In: Germanistische Werkstatt 6, S. 81-92.

Kovács E. (2009), Anglizismen im Deutschen: eine Gefahr oder natürliche Erscheinung? In: Germanistische Studien VII, S. 181-190.

Lewandowski Th. (1994), Linguistisches Wörterbuch, Heidelberg.

Lipczuk R. (2012), Motive der Bekämpfung der Fremdwörter im Deutschen. In: Zeitschrift des Verbandes Polnischer Germanisten, 2012, Zeszyt 2 (2012), Kraków, S. 177-192.

Sadziński W. (2014), „Anglizismenwucherung “ im Nachrichtenmagazin „,Der Spiegel“. In: Kaczmarek D., Makowski J., Michoń M. (Hrsg.): Texte im Wandel, Łódź, S. 169-178.

Sick B. (2009), Er designs, sie hat recycled, und alle sind chatting. In: Sick, B.: Der Dativ ist dem Genitiv sein Tod. Ein Wegweiser durch den Irrgarten der deutschen Sprache. Folge 1, S. $151-153$.

Miller D. (2003), Zum Vergleich der deutschen Anglizismusdebatte in der Medienöffentlichkeit und Linguistik. In: Lingua ac Communitac Vol. 13, S. 43-54.

Spieß C. (2011), Die sprachlich-diskursive Konstitution von Weltanschaunn und Weltbild im Stammzellendiskurs durch Lexik, Metaphorik und Argumentationsmuster. In: Tekst i Dyskurs/Text und Diskurs 4, S. 133-156.

Spitzmüller J., Warnke I. H. (2011), Diskurslinguistik. Eine Einführung in Theorien und Methoden der transtextuellen Sprachanalyse, Berlin.

Yang W. (1990), Anglizismen im Deutschen: am Beispiele des Nachrichtenmagazins Der Spiegel, Tübingen. 


\title{
Marcelina Kałasznik
}

\section{STAR, CELEBRITY, VIP - ON THE EFFECT OF THE ENGLISH LANGUAGE ON THE SEMANTIC FIELD OF TERMS OF KNOWN PEOPLE IN GERMAN}

\author{
(Summary)
}

It is clear that no language exists in isolation and through personal encounters, contact between languages results in lexical borrowings. The influence of one language on another can be perceived in a positive way - as an enrichment of the lexical language of the recipient, or a negative way - for example, as an attempt to replace native lexical units with those of foreign origin. In this article we have made an attempt to analyze three individual personal designations (Celebrity, Star, VIP) borrowed by German from English. This article aims to discuss examples of lexemes, taking into account such aspects of their adaptation and integration into the German language as semantics, frequency of occurrence and formative productivity.

Keywords: Anglicisms, personal designations, designations of known persons. 